\title{
Human endogenous oxytocin and its neural correlates show adaptive responses to social touch based on recent social context
}

\author{
Linda Handlin $\dagger^{1}$, Giovanni Novembre $\dagger^{2}$, Heléne Lindholm ${ }^{1}$, Robin Kämpe ${ }^{2,3}$, India \\ Morrison* 2,3
}

${ }^{1}$ Department of Biomedicine, School of Health Sciences, University of Skövde, 54128 Skövde, Sweden

${ }^{2}$ Center for Social and Affective Neuroscience, Department of Biomedical and Clinical Sciences, Linköping University, 58183 Linköping, Sweden

${ }^{3}$ Center for Medical Image Science and Visualization (CMIV) Linköping University Hospital, 58185 Linköping, Sweden

*Correspondence to: india.morrison@liu.se.

$\dagger$ The first and second authors contributed equally to this work 


\begin{abstract}
Both oxytocin (OT) and touch are key mediators of social attachment. In rodents, tactile stimulation elicits endogenous release of OT, potentially facilitating attachment and other forms of prosocial behavior, yet the relationship between endogenous OT and neural modulation remains unexplored in humans. Using serial sampling of plasma hormone levels during functional neuroimaging, we show that contextual circumstances of social touch facilitate or inhibit not only current hormonal and brain responses, but also calibrate later responses. Namely, touch from a romantic partner enhanced subsequent OT release for touch from an unfamiliar stranger, yet OT responses to partner touch were dampened following stranger touch. Hypothalamus and dorsal raphe activation reflected plasma OT changes during the initial interaction. In the subsequent social interaction, OT modulation depended on the previous interaction, mediated by precuneus and parietal-temporal cortex pathways, including a region of medial prefrontal cortex that also covaried with plasma cortisol. These findings demonstrate that hormonal neuromodulation during successive human social interactions is adaptive to social context, and they point to mechanisms that flexibly calibrate receptivity in social encounters.
\end{abstract}

\title{
Introduction
}

A hug from a friend, a caress from a lover, the secure embrace of a parent: touch is a predominant sensory channel for bolstering human connection and emotional attachment. The neural mechanisms supporting this vital role of touch are not fully understood, but there is evidence from rodents that tactile stimulation in social interactions can act as a major trigger for endogenous release of the neuropeptide oxytocin (OT) $(1,2)$, which has been implicated in social attachment $(3,4)$. It has therefore been suggested that social tactile stimulation such as affectionate stroking may elicit endogenous OT release in adult humans (5-8).

In this perspective, afferent tactile stimulation initiates a cascade of modulatory responses in the brain, mediated by specific neural populations in the hypothalamus. It is wellestablished that magnocellular oxytocin neurons in the paraventricular nucleus (PVN) of the hypothalamus send projections to forebrain and cortical regions mediating behavioral, olfactory, and somatosensory signaling in the brain (9-12). For tactile stimulation, this 
signaling may rely on a recently-discovered population of parvocellular OT neurons in the rat PVN selective for particular forms of affiliative touch (1). OT released into the bloodstream via a PVN-pituitary pathway modulates the action of vasculature and smooth muscle (13), notably during processes surrounding reproductive behavior in both sexes, as well as parturition and lactation in females $(14,15)$.

OT's broad role in parental nurturance and affiliative behavior may reflect a functional extension of its influence on these core reproductive and maternal behaviors, many of which rely on sensory cues such as touch and olfaction, encompassing even cross-species interactions (16-18). Many OT-relevant sensory stimuli likely involve central pathways of OT in the brain, but peripheral mechanisms of release can also be triggered by stimulation of the genitals, the nipples, or the vagal nerve. However, despite evidence for afferent-driven central OT release, it is also acknowledged that OT-mediated social neuromodulation is highly dependent on factors such as social familiarity (19-21) and current physiological state $(22,23)$, though this has been little explored for human endogenous OT. For example, endogenous OT can mediate prosocial allogrooming behavior in mice (24), but OT and OT receptor genotype have also been shown to play a role in antagonistic social behaviors such as defense of offspring (25) and aggression (26,27). Further, optogenetic manipulation of the same PVN OT neurons in freely-behaving mice can result in either prosocial or antagonistic behavior (28). These observations suggest that OT's role in social behavior is multivalent and situational $(29,30)$. Proposed functional roles for OT as selectively modulating affiliative social relationships (31) or maintaining allostatic stability (22) accommodate such differential, context-dependent effects. Nonetheless, a marked gap remains to be bridged between stimulus-driven and context-dependent frameworks in charting the neural mechanisms of OT effects on social behavior.

In humans, it is not known whether endogenous OT changes and their neural correlates during social interactions may be selectively modulated by specific contextual conditions. However, the influence of exogenous, intranasal OT (IN-OT) administration on behavioral and neural outcomes have been widely studied with regard to social stimuli $(e g, 33,34)$. INOT has shown varying effects on social outcome measures such as face processing (35-37), empathy (38), romantic bonding (39-42), and romantic touch (43). Neuroimaging studies of IN-OT manipulations indicate that many relevant changes occur at the cortical level $(44,45)$, 
suggesting more complex modulatory pathways than is implied by a stimulus-driven model focused on subcortical signaling. However, as there is uncertainty surrounding the mechanisms of action of IN-OT and its degree of equivalence to endogenous release (46-49; but see 50), it is crucial to investigate endogenous OT changes and their neural correlates in humans for a fuller understanding of the relevant mechanisms, as well as their potential limits and parameters.

In this study, we therefore combined serial sampling of plasma OT with functional magnetic resonance imaging (fMRI), to examine whether social interactions involving touch can evoke endogenous changes in plasma OT in human females. We predicted that touch from a socially familiar person (a romantic partner) would evoke greater endogenous OT changes than touch from an unfamiliar person (a nonthreatening stranger), thus allowing investigation of the neural responses associated with this difference. We predicted higher activations in hypothalamus and other key regions associated with oxytocin modulation and receptor expression in different species (such as amygdala, medial prefrontal cortex, and cingulate). Beyond this, we also explored whether any such changes would be modulated by whether the partner touched before the stranger, and vice-versa (Fig S1). To investigate this key temporal aspect of context, we compared OT and neural responses to partner and stranger touch across two successive interactions, one in which the stranger preceded the partner, and one in which the partner preceded the stranger. These social-contextual factors allowed us to identify brain regions in which hemodynamic responses changed as a function of endogenous OT levels over time, revealing conditional, OT-mediated engagement of subcortical and cortical systems.

\section{Results}

42 females in committed heterosexual romantic relationships of at least one year (mean age 24.6 years, $\mathrm{SD}=4.6$, Table $\mathrm{S} 1,6$ ), participated in the study with their male partners (mean age 26.8 years, $\mathrm{SD}=6.0$ ). Female participants were included if they were between $19-40$ years, were not pregnant or breastfeeding, were free from estrogen-based contraceptive use, and were not undergoing current or recent hormone therapy. The female in the couple participated in fMRI scanning and provided plasma samples, while the male partner administered touch during the experiment. Ethical approval was obtained from the Regional Ethical Review 
Board in Linköping, Sweden. All participants gave informed consent in accordance with the Declaration of Helsinki and were compensated at 400 SEK ( 45 USD)/h.

Approximately $1 \mathrm{~h}$ prior to data collection, an indwelling magnet-safe catheter was inserted into the cubital vein of the female participants' left arm (Fig 1A; Supplementary Methods). During the functional magnetic resonance imaging (fMRI) session, 9 serial blood samples were collected through the catheter using vacutainer tubes, three "baseline" samples (pre-, intermediate, and -post; samples $(1,5,9)$ and 3 serial samples for each functional run $(2-4,6$ 8). The two 7-min functional runs were separated by $\sim 27 \mathrm{~min}$, allowing OT levels to return to baseline at sample 5 (Fig 1B). Baseline OT levels (first and intermediate) preceding each functional run did not differ significantly between participants who were touched by their partner compared to the stranger in the first run (first: $\mathrm{t}=1.801, \mathrm{df}=25, \mathrm{p}=0.084$; intermediate: $\mathrm{t}=1.1843, \mathrm{df}=25, \mathrm{p}=0.077$; effect size Cohen's $\mathrm{d}=.71$, two-tailed, allocation ratio .85$)$. The experimental paradigm implemented a 2x2x2 factorial design: "toucher" (partner/stranger, blocked by run), "encounter order" (reflecting the counterbalancing of partner or stranger in the each of the two encounters), and "location" of the touch (arm or palm, pseudorandomized throughout each of the two 7-min functional runs). Each run consisted of twelve 12-s touch stimulation trials, with arm and palm stimulation pseudorandomized within runs, with a jittered 21-30s intertrial interval.

Touch stimulation was delivered manually in the scanning environment by the male partner ("partner") and a male experimenter ("stranger"), who was briefly introduced to the participant before the experiment and was rated as trustworthy (mean +3.96 on a -10 to +10 scale) after the experiment. Caressing strokes were delivered to the right dorsal arm and palm, with timing and touch site guided by auditory cues via headphones. The person delivering touch (male partner/stranger) was positioned beside the scanner bore on the right side of the participant. A nurse and an assistant collecting blood samples were positioned beside the scanner bore on the left side of the participant. In the "initial encounter" (the first of two runs), participants either received touch from their partner or the stranger, and vice-versa in the "second encounter" ( $\mathrm{N}=23$ and $\mathrm{N}=18$, respectively), thus allowing investigation of any conditional influence by the initial encounter upon the second encounter. 


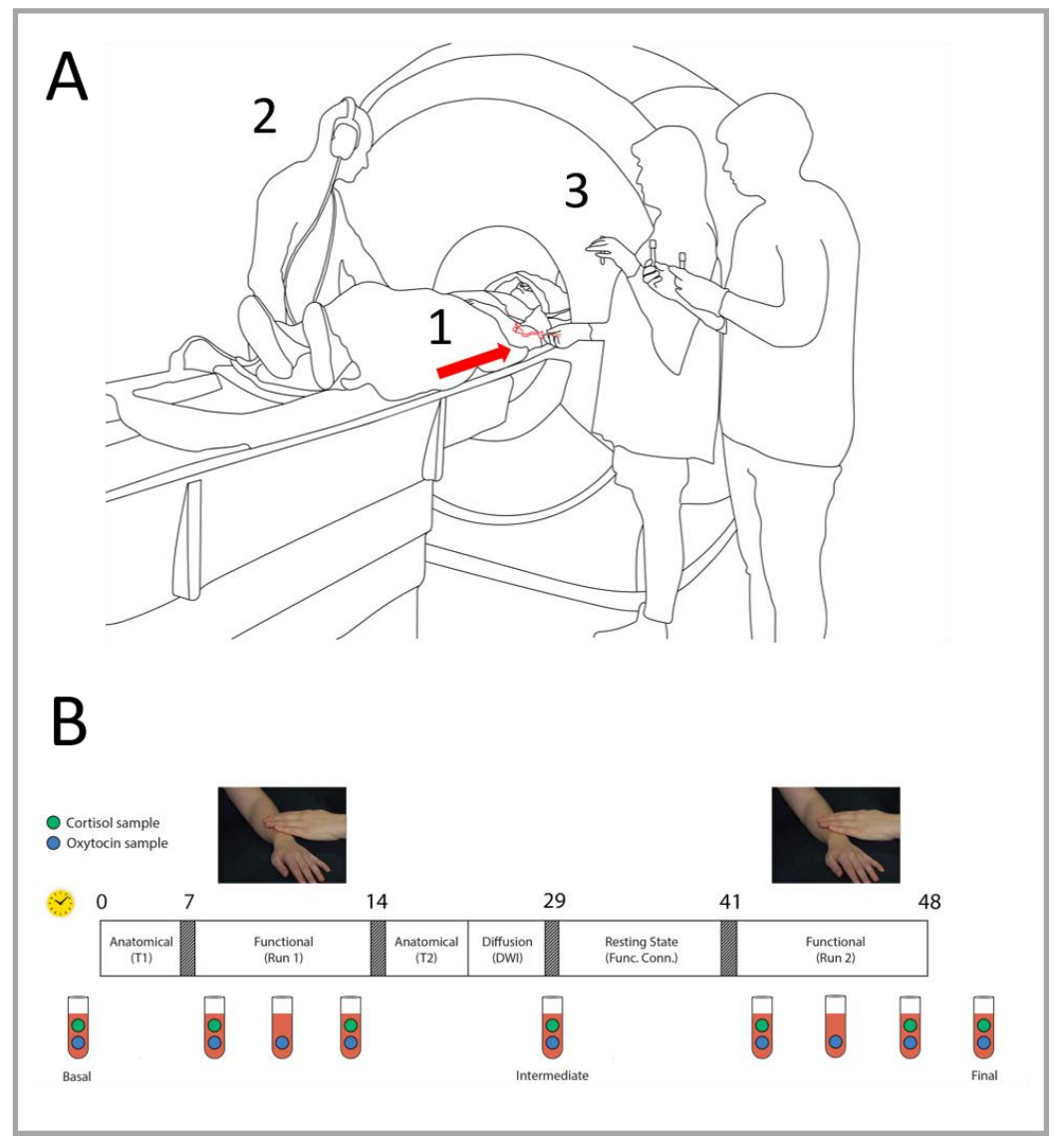

Figure 1. A. fMRI experiment setup. 1) Indwelling catheter in female participant's left arm (arrow); 2) participant's male partner or unfamiliar stranger caressed participant, following audio prompts; 3) serial blood samples were collected from the catheter. B. Structure of fMRI experimental session with serial sampling of plasma oxytocin and cortisol. Rectangle depicts time course of experiment, with approximate elapsed minutes shown above (yellow clock symbol). Two functional runs with partner and stranger touch, in counterbalanced order, were separated by 27 min. 3 baseline OT samples $(1,5,9)$ and 3 serial samples were collected for each run (2-4, 6-8). Clock symbol indicates time in minutes. Blue dots in the vial symbols indicate oxytocin samples, green dots indicate cortisol samples. The first functional run was preceded by acquisition of an anatomical (T1-weighted) image, while between functional runs additional anatomical and functional scans were acquired: T2-weighted anatomical image, diffusion-weighted imaged, and resting-state.

In the last $7 \mathrm{~s}$ of each trial, the participant rated the pleasantness of the touch on a visual analogue scale (VAS), anchored with "most unpleasant imaginable touch" on one extreme and "most pleasant imaginable touch" on the other. Responses were made with the right hand 
using a response pad system (4-Button Diamond Fiber Optic Response Pad, Current Designs). Partner touch was rated as more pleasant than stranger touch $(F=1,34, p<0.001$; Cohen's $d=$ 2.15) with a main effect of higher ratings for arm $(F=1,34, p=0.003$; Cohen's $d=.22)$. A significant three-way interaction $(\mathrm{F}=1,34, \mathrm{p}=0.037)$, was driven by lower ratings for stranger touch on the palm compared to stranger touch on the arm, for participants who received stranger touch first, $(\mathrm{t}=16, \mathrm{p}=0.007)$.

After the MR session, female participants evaluated how relaxing they had found partner and stranger touch, as well as how attractive and trustworthy they found the stranger. Mean partner touch ratings were rated as more relaxing than stranger touch $(\mathrm{P}<0.00001)$, but stranger palm was rated as less relaxing than stranger arm touch $(\mathrm{P}=0.05)$. The stranger was rated as positively trustworthy, and attractiveness was near a neutral midpoint (see Supplementary Results). For participants in the stranger first group, the smaller the difference between pleasantness ratings for stranger and partner touch during the imaging session, the more relaxing participants rated stranger touch afterwards $(r=-.70, P=0.004)$. Withinsession pleasantness ratings also predicted post-session evaluations of relaxation for partner and stranger touch independently (partner $\mathrm{r}=0.83, \mathrm{P}=0.0002$; stranger $\mathrm{r}=0.80, \mathrm{P}=0.001$ ). For participants starting with partner touch, evaluation of relaxation for partner touch correlated with how trustworthy participants rated the stranger $(\mathrm{r}=.77, \mathrm{P}=0.002)$.

Both female and male participants provided questionnaire data. Couple Satisfaction Index (CSI) scores indicated that both female and male participants were satisfied with their relationships (females: mean=139.8, $\mathrm{sd}=17.2$, males: mean=139.6, $\mathrm{sd}=15.2$ ). State-Trait Anxiety Inventory (STAI) scores indicated that no participants demonstrated clinically significant symptoms of anxiety (females STAI-S mean=35.6, sd=9.7; female STAI-T mean=40.3, sd=9.8; males STAI-S mean=31.6, sd=5.7; males STAI-T: mean=36.2, sd=6.7). Participants' assessments of relationship quality correlated with their partners' ( $\mathrm{r}=0.57$, $\mathrm{p}=0.0003$ ). Participants' trait anxiety correlated inversely with the both the participant's and her partner's assessment of relationship quality $(r=-0.40, \mathrm{p}=0.01$ for participant, $\mathrm{r}=-0.39$, $\mathrm{p}=0.01$ for partner).

Plasma OT responses depend on who touched first. Plasma samples were extracted using acetonitrile precipitation and OT concentrations determined using ELISA (Enzo Life 
Sciences; sensitivity $>15.0 \mathrm{pg} / \mathrm{ml}$, intra-assay precision $10.2-13.3 \% \mathrm{CV}$, inter-assay precision 11.8-20.9\% CV), and the order effect was assessed by a mixed linear model including all OT samples from participants with a complete sample series ( $\mathrm{N}=27$; Table s2). Participants' maximum (peak) plasma OT concentrations for each functional run were entered into a mixed linear model alongside baseline, intermediate, and final values using SPPS version 25. The model included the within-subject factors timepoint (position of sample in series) and order (partner or stranger first). The results revealed that order influenced OT changes (Fig 2A), with a main effect of order of person delivering touch (partner or stranger first/second; $F=4,577, P=0.042)$; as well as of timepoint, $(F=8.183, p<0.0001)$, but no interaction.

When the partner's touch occurred during the initial encounter, the mean of the maxima (peaks) of OT concentrations sampled during the functional run increased from baseline, whereas there was no such increase for stranger touch (partner: $\mathrm{t}=-2.26, \mathrm{df}=13, \mathrm{P}=0.041$, $\mathrm{sd}=31.69$; stranger: $\mathrm{df}=12, \mathrm{P}=0.114, \mathrm{sd}=27.45$ ). Peak OT was marginally lower for stranger compared to partner over the whole functional run but did not differ significantly, although the effect size was large $(\mathrm{t}=1.81 ; \mathrm{df}=25, \mathrm{P}=0.082$, effect size $\mathrm{d}=.97$, two-tailed, allocation ratio .85; Fig 2A). During the second encounter, peak OT was higher for stranger compared to partner touch $(\mathrm{t}=2.37, \mathrm{df}=25, \mathrm{P}=0.026)$, yet with significant increases from baseline for each (partner: $\mathrm{t}=3.265, \mathrm{df}=13, \mathrm{P}=0.006$, $\mathrm{sd}=14.9$; stranger: $\mathrm{t}=3.258, \mathrm{df}=12, \mathrm{P}=0.007$, $\mathrm{sd}=4.77$; Fig 2A). OT for partner touch in the second encounter was also significantly lower compared to partner touch in the initial encounter $(\mathrm{t}=2.63, \mathrm{df}=24, \mathrm{P}=0.015)$.

Individuals' mean OT in the initial encounter predicted mean OT levels in the second (partner-stranger $\mathrm{r}=0.97, \mathrm{P}<0.001$; stranger-partner $\mathrm{r}=0.57, \mathrm{P}=0.03$; Fig $2 \mathrm{~B}$ ). No correlation was found between mean OT levels and mean pleasantness ratings (all Ps > 0.05), but OT changes for stranger in the initial encounter predicted post-session ratings of his trustworthiness ( $\mathrm{P}=0.009$; Supplementary Results). 
bioRxiv preprint doi: https://doi.org/10.1101/2021.04.08.438987; this version posted April 9, 2021. The copyright holder for this preprint (which Handlin, Novembre et al was not certified by peer review) is the author/funder. All rights reserved. No reuse allowed without permission.
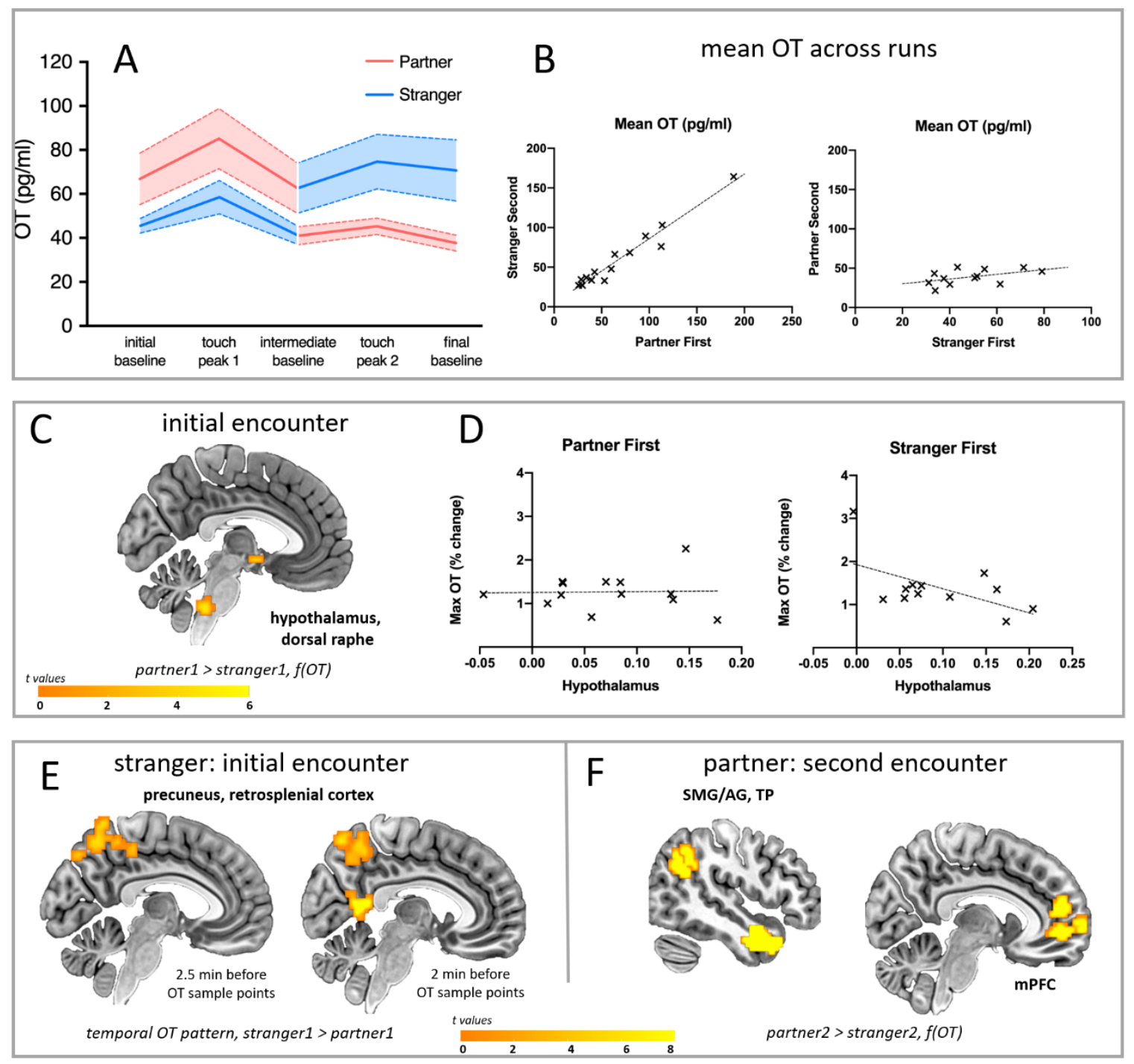

Fig 2. Endogenous oxytocin (OT) changes and covariant brain responses. A. In the initial encounter, plasma OT increased for partner (red; $\mathrm{P}=0.041$ ) but not stranger (blue; $\mathrm{P}=0.114$ ), compared to the respective baselines. In the second encounter OT increased for both partner $(\mathrm{P}=0.006)$ and stranger $(\mathrm{P}=0.007)$ touch, with higher mean peak values (maxima) for stranger compared to partner $(\mathrm{P}=0.026)$. OT for partner touch in the second encounter was lower compared to partner in the initial encounter $(\mathrm{P}=0.01)$. B. Mean OT in the initial encounter predicted mean OT in the second (partner first: $\mathrm{r}=0.97, \mathrm{p}<0.001$; stranger first: $\mathrm{r}=0.57, \mathrm{P}=$ 0.03). C. BOLD changes in hypothalamus and dorsal raphe nuclei covaried with peak OT levels (greatest \% change from baseline) in the initial encounter, in the contrast partner > stranger. D. OT-BOLD covariance in hypothalamus and dorsal raphe (not shown) were driven by a greater 
decrease for stranger touch during the initial encounter (scatterplots show beta values

unweighted by OT covariate). E. The temporal pattern of OT-BOLD changes in bilateral

precuneus preceded sampling by $2.5 \mathrm{~min}$, with retrosplenial cortex also emerging at $2 \mathrm{~min}$, with greater covariance for stranger touch during the initial encounter. F. BOLD changes in

SMG/AG, TP, and mPFC covaried with peak OT levels (greatest \% change from baseline) in the contrast partner $>$ stranger in the second encounters $(\mathrm{P}<0.002)$. BOLD $=$ blood-oxygen-leveldependent, $I T G=$ inferior temporal gyrus, SMG/AG = supramarginal gyrus/angular gyrus, $T P$ = temporal pole, $m P F C=$ medial prefrontal cortex, $f(O T)=$ as a function of OT .

\section{Subcortical and cortical activations reflect aspects of OT differences and contextual shift.} Hemodynamic correlates of endogenous OT changes during these social touch interactions were measured using functional magnetic resonance imaging (fMRI), acquired using a 3.0 Tesla Siemens scanner (Prisma, Siemens) with a 64-channel head coil. Preprocessing and statistical analysis of MRI data were performed using Analysis of Functional Neuroimages (AFNI) statistical software (version 19.1.12). fMRI results were derived from a linear mixedeffects modeling analysis (3dLME) with percent change values of OT as covariates, at a voxel-wise $\mathrm{P}$-value threshold of $\mathrm{P}=0.002$, and a cluster-size threshold of alpha $=0.05$ familywise-error (FWE; $\mathrm{N}=35$ complete functional datasets). See Supplementary Methods for details of modeling and analysis.

During the initial encounter, individual changes in plasma OT levels (greatest \% change from baseline) for partner compared to stranger touch covaried with blood-oxygen-leveldependent (BOLD) signal changes in the hypothalamus (MNI 5, -5, -8; Fig 2C; Table s3; significant at uncorrected threshold of $\mathrm{p}<0.002$, with a subset of 15 voxels surviving stricter cluster correction in a small volume region-of-interest analysis; see also Supplementary Results). BOLD in dorsal raphe nuclei also covaried with plasma OT in a whole-brain analysis (MNI -11, -47, -41; 7, -44, -38; Fig 2C). In each of these regions, the difference between partner and stranger covariance showed greater negative correlation with OT levels during stranger touch (hypothalamus-mean OT: $r=-.79, p=0.001$; raphe-mean OT: $r=-.75$, $\mathrm{p}=0.003$; Fig 2F). Hypothalamus and dorsal raphe activation was correlated during the first encounter with the stranger $(r=.69, p=0.009$; Fig $2 F)$. 
During the second encounter, OT covaried more strongly with partner than stranger touch in the right temporal pole (TP; MNI 40, 19, -32) and supramarginal and angular gyri (SMG/AG; MNI 49, -53, 34), extending into posterior superior temporal cortex (pSTS), alongside medial prefrontal cortex (mPFC) and adjacent anterior cingulate cortex (ACC; MNI $-11,49,1$; Fig 2E). These activations partly explained an interaction between identity of person delivering touch and OT levels in $\mathrm{mPFC} / \mathrm{ACC}$, alongside right TP and SMG/AG $(\mathrm{P}<0.002 ; \mathrm{s} 2$; Tables s3, 6). Further, an anterior inferior temporal gyrus (ITG) cluster covaried with touch pleasantness. Specifically, ITG activation covaried with the difference between partner and stranger touch pleasantness ratings when partner touched first (peak cords, $\mathrm{p}<$ 0.005; Fig 2C), with individuals that showed higher activation during the first encounter compared to the second also showing the largest rating difference. When the stranger delivered touch in the second encounter, ITG activation increased with touch pleasantness during the run $(r=0.65, p=0.003$; Fig 2D), and correlated with greater self-reported relaxation from stranger touch after the run $(r=0.74, p=0.003)$. For partner touch in the second encounter, ITG negatively correlated with post-session stranger attractiveness evaluations $(\mathrm{r}=-0.76, \mathrm{p}=0.002)$.

We also explored any temporal patterns of the brain-plasma OT relationship using a novel retrospective modelling approach which identified overall patterns in regional BOLD changes that preceded OT sample collection points by various time lags. To do this, we applied a regressor based on linear interpolation of OT sample datapoints, convolved with the canonical hemodynamic response function (HRF; see also Supplementary Methods). This analysis revealed that precuneus activation tracked the pattern of increases and decreases in plasma OT. Further, it did so more strongly for stranger compared to partner touch in the initial encounter, emerging 2.5 min prior to sampling (MNI -8, -62, 67; 10, -65, 58), with retrosplenial (MNI 10, -53, 10) and mPFC cortices also emerging 2 min prior (Fig 2E; Table s4). The mPFC cluster also showed an interaction between identity and order of person delivering touch with OT levels ( $\mathrm{P}<0.002$; Fig 2E).

Plasma cortisol correlates with mPFC modulation. Plasma cortisol levels were determined using the Cortisol Parameter Assay Kit according to the manufacturer's recommendations (R\&D Systems, Minneapolis, Minnesota, USA) (sensitivity $0.071 \mathrm{ng} / \mathrm{mL}$, precision 10.4\%). 
Cortisol analysis serum samples were diluted 60 times preceding analysis. Pretreatment steps of the serum samples resulted in a dilution factor of three and the pretreated serum samples required an additional 20-fold dilution in Calibrator Diluent RD5-43.

A similar mixed linear model (including all seven cortisol samples; $n=26$ ) as the one used for OT revealed a significant main effect of timepoint, $(\mathrm{F}=9.527), \mathrm{P}<0.001)$ with an interaction between timepoint and order $(\mathrm{F}=4.415, \mathrm{P}<0.001)$. Post-hoc t-tests revealed that this interaction was likely driven by higher basal cortisol levels for those touched by the stranger first compared to partner first (partner vs stranger run $1, \mathrm{t}=-1.991, \mathrm{df}=24, \mathrm{P}=0.058$, sd), alongside greater increases for the first two samples of the session (pre-touch and sample 1 in run 1, pre-touch: $\mathrm{t}=-1.991, \mathrm{df}=24, \mathrm{P}=0.058 ; 1 \mathrm{~min}$ run $1: \mathrm{t}=-2.051, \mathrm{df}=24, \mathrm{P}=0.051$ ).

Activation for initial partner touch in the hypothalamus region of interest showed a correlative relationship with cortisol for stranger in the second encounter $(r=.79, p=0.007)$, which correlated with dorsal raphe in the second encounter $(\mathrm{r}=.73, \mathrm{p}=0.01)$.

Time of day (i.e. sessions beginning at 9:00, 12:00 or 15:00) did not have a significant effect on the participant's basal cortisol levels. Cortisol and OT showed no direct correlative relationship, contrary to our original prediction, but cortisol levels covaried with BOLD for partner in the initial encounter in regions including mPFC (MNI -11, 46, 10; Fig. 2E; Table s7), which showed a subset of OT-sensitive voxels (Fig 2F). 

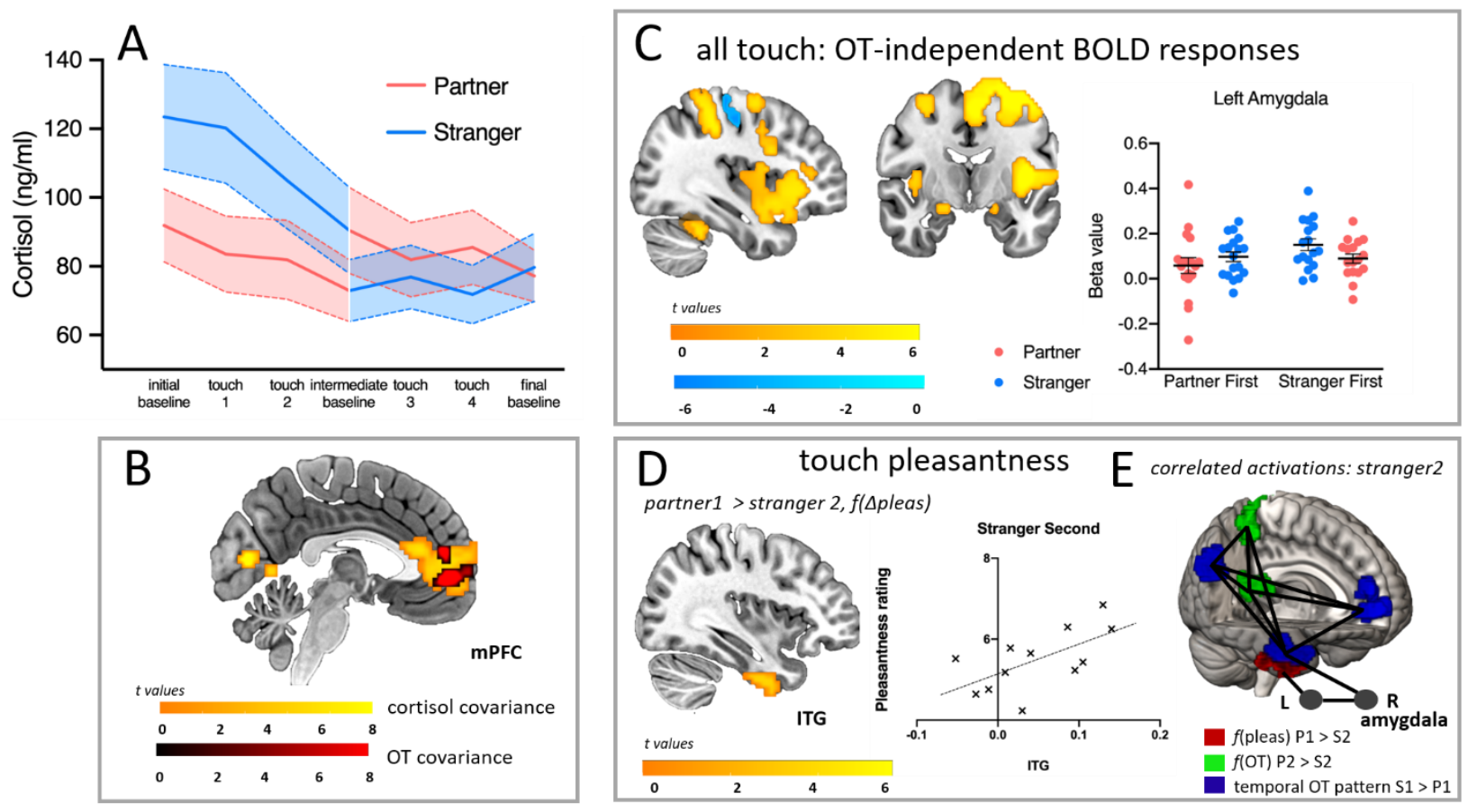

Fig 3. Plasma cortisol and covariant brain responses, and OT-independent brain activation. A. Plasma cortisol was sampled at the three "baseline" points and twice per functional run. Participants starting with stranger touch showed marginally higher initial cortisol levels $(\mathrm{P}=0.058)$, decreasing over the session. B. BOLD signal change in regions including mPFC covaried as a function of cortisol levels, with partner > stranger $(\mathrm{P}<0.002)$, and a subset of mPFC voxels covarying with both OT (partner > stranger, second encounter) and cortisol (partner > stranger, initial encounter). C. BOLD changes in somatosensory and insular cortices, as well as bilateral amygdalae, across all touch conditions, independently of familiarity of person delivering touch, order, and OT levels (all Ps <0.002). Left amygdala was sensitive to partner-stranger differences (main effect of toucher, $\mathrm{F}(1,16)=5.8, \mathrm{P}=$ 0.028), with stranger $>$ partner in the initial encounter $(P=0.02)$. D. ITG was sensitive to differences in partner and stranger touch pleasantness ratings ( $\Delta$ pleas), reflected in decreased BOLD covariance in ITG $(\mathrm{P}<0.005)$ during stranger touch in the second encounter (mean pleasantness partner $>$ stranger, $\mathrm{P}$ $<0.001)$. E. Specific changes in correlative relationships among cortical regions-of-interest and amygdalae for stranger touch in the second encounter. Mean beta values in left amygdala correlated with those in with TP and ITG for stranger touch (amygdala-TP r = 0.62, $\mathrm{p}=0.006$; amygdala-ITG $\mathrm{r}=$ 0.73, $\mathrm{p}=0.001$ ), alongside positively correlative relationships among precuneus, retrosplenial cortex, 
SMG/AG, TP, and mPFC clusters (all Ps >0.001; see Suppl Mat). BOLD = blood-oxygen-level-

dependent, $I T G=$ inferior temporal gyrus, $S M G / A G=$ supramarginal gyrus/angular gyrus, $T P=$ temporal pole, $m P F C=$ medial prefrontal cortex, VAS = visual analogue scale (touch pleasantness); $f(O T)=$ as a function of OT.

Regional brain responses independent of OT changes. BOLD responses in several key regions were independent of plasma OT changes (Fig 2A-C), suggesting an absence of direct, covariant modulation by endogenous OT. Somatosensory and posterior insular cortices showed general responses to touch in a conjunction across all touch conditions (Fig2C; Table s6) and activation here did not significantly covary with OT levels in the covariate analyses. This was also the case for posterior insula-parietal opercular activations selective for hairy skin stimulation (arm vs palm stroking; Supplementary Materials). A subtype of C afferent nerve with a preference for gentle, caresslike stimulation is found predominantly in skin containing hair follicles, stimulation of which selectively activates posterior insular cortices. However, the present findings do not support any direct, specific covariant modulation of CTafferent-rich skin by endogenous OT.

Conjunction analysis also revealed common activations for partner touch in the caudate nucleus in both the initial and second encounters, regardless of order, suggesting possible facilitation of dorsal striatum by partner touch in the absence of a specific, direct relationship with OT changes. Similarly, touch activated the bilateral amygdalae in a general fashion in a conjunction across all encounters (Fig 3D,E; Table s5), with left amygdala selective for stranger touch (main effect stranger: $\mathrm{F}(1,16)=5.8, \mathrm{P}=0.028$ ), particularly in the first encounter (stranger $>$ partner $\mathrm{P}=0.02$ ).

Correlative relationships among regions-of-interest. Linear correlations were explored among mean beta values in the cortical regions defined by covariance with endogenous OT and/or subjective ratings (precuneus, retrosplenial cortex; SMG/AG, TP; ITG), and including amygdalae and bilateral caudate clusters for each group separately ("partner first" and "stranger first"). This revealed consistent significant correlations between these regions of interest across runs, suggestive of network-level relationships (all Pearson correlation coefficients $>0.58$, all $P$ s $<0.01$, 2-tailed; summarized in Fig S2). Correlations were more 
extensive for the stranger touch group, both within and between runs (Fig S2). A sparser set of positively correlative relationships was seen for the partner first group, mainly among precuneus, retrosplenial cortex, SMG/AG, TP, and mPFC clusters, with a negative correlation between precuneus and TP from the first to the second run (Fig S2). Left amygdala activation correlated specifically with the retrosplenial cluster when partner touched first, and an ITG$\mathrm{mPFC}$ correlation was also specific to this condition. During subsequent stranger touch, left and right amygdalae correlated with ITG and TP, respectively (Fig 2E).

\section{Discussion}

A touch-mediated social interaction with a familiar partner is embedded in a series of many events over the course of the relationship. On the other hand, an interaction with a person one has never met can lay the groundwork for future interactions. The present findings shed light on the potential contribution of OT neuromodulation in such flexible, context-dependent social responses. They demonstrate not only that social touch interactions in humans can influence endogenous OT and brain responses, but that the familiarity of the person delivering touch and the recent history of social interaction modulate these changes in a conditional manner.

In the initial touch encounter, the preferential increase in plasma OT levels for the partner is consistent with familiarity-dependent effects of OT in rodents (24). This basic partnerstranger difference was reflected in hypothalamus covariance as a function of individual peak plasma OT levels. This is in accord with the mammalian neuroanatomy of central OT release, in which hypothalamic nuclei, particularly the PVN, synthesize OT and stimulate its release in the brain $(9-12,51)$. In rats, stroking touch increases Fos protein expression in PVN (52), and recent evidence from freely-interacting female rats indicates that a population of parvocellular OT neurons in PVN is selectively tuned to social touch stimulation (1). In the present study, BOLD changes in dorsal raphe nuclei also covaried with plasma OT (Fig 2C), possibly reflecting descending influence on seratonergic circuits (53) in modulating touch signaling from the periphery. Hypothalamus and dorsal raphe activation showed a negative relationship with mean OT when stranger touched first (Fig 1), indicating that these clusters have a negative, context-dependent influence on endogenous OT. Such an influence may not hold for, or may be buffered by, partner touch. 
While the initial encounter provides a glimpse into processes which may calibrate subsequent endogenous OT release, the second encounter opens a window onto contextdependent facilitatory and inhibitory effects of those processes. Participants whom the partner had touched in the initial encounter showed a robust OT response for stranger touch in the second encounter. In light of previous evidence for cumulative effects of central OT exposure over repeated interactions with specific individuals (15), as well as the established contextsensitivity of OT effects in nonhuman mammals (23), this order-dependence of OT responses indicates a critical influence of social interaction history and current physiological state. In contrast, participants receiving stranger touch in the first encounter displayed a significantly lower OT increase during subsequent partner touch, as compared to partner touch in the initial encounter. This may reflect a sluggish return from any inhibitory processes involved in the initial stranger touch.

This diminished, potentially recovery-related response during partner touch in the second encounter was accompanied by BOLD changes in superior temporal and inferior parietal regions. These regions have been implicated in individual intranasal-OT studies of partnerstranger interactions (41-43) as well as in meta-analyses of intranasal-OT fMRI activation $(44,45)$, in multisensory and contextual integration processes (54), and even in tactile foot massage (6). These activations may thus reflect OT-mediated updating of contextual information, possibly enhancing the salience of incoming sensory signals (55-58) following the initial encounter with the stranger. For example, the covariant relationship of ITG with hedonic and social evaluation ratings and amygdala activation, especially for partner touch in the second encounter (Fig 2D), indicates a potential role for this region in maintaining positive receptivity to touch following contextual shifts. Such biased modulation suggests neural gain-control mechanisms that operate in conjunction with endogenous OT, which can facilitate or dampen hormonal and neural responses in successive social interactions.

Central effects of IN-OT have consistently been found $\sim 45$ min post-administration (6961), but there is less direct evidence about the timecourse of central endogenous OT release into the periphery in humans. We therefore developed an exploratory regressor based on the serial pattern of individuals' OT levels, convolved with the HRF. This allowed us to look "backwards" from the plasma OT sampling to any preceding hemodynamic activation that covaried with them. The pattern-covariant engagement of the precuneus at 2.5 minutes 
preceding sampling, and of precuneus, retrosplenial cortex, and $\mathrm{mPFC}$ at 2 minutes, is within the frame of the half-life of OT in blood (62) and may reflect events surrounding central OT release. Retrosplenial cortex projects to mPFC (63), while precuneus is functionally connected to the SMG/AG region activated for partner during the second encounter (64). INOT administration can also induce changes in functional connectivity between precuneus and AG (64). This is the first evidence of selective network modulation of precuneus by endogenous OT, and implies that precuneus, retrosplenial cortex, and mPFC may act as arbiters of activation in parietotemporal and limbic networks, potentially influencing responses to social touch via contextual integration and affect regulation processes. These OT-dependent neural dynamics may play a critical role in calibrating social receptivity, especially over multiple social encounters. It is not possible to determine the directness or direction of influence from these exploratory findings, but the temporal directionality suggests that cortical processing precedes plasma changes.

OT has been also implicated in stress regulation via corticotropin-releasing-hormone (CRH)-cortisol pathways, and may act as a physiological regulator of acute stress-related responses (65-69). We therefore collected serial measurements of plasma cortisol alongside OT. However, despite initially higher plasma cortisol levels for stranger than partner, cortisol and OT showed no direct relationship. Plasma cortisol levels covaried with BOLD for partner in the initial encounter in several regions, including a mPFC cluster which contained a subset of OT-sensitive voxels (Fig 2B). The mPFC is associated with limbic circuitry regulating bodily responses that inform emotional processing (70). Further, both mPFC, implicated in cortical-amygdala signaling following IN-OT (73), and superior temporal gyrus (STG), implicated across a range of multisensory integration, including touch (71-73) decreased for initial stranger touch as a function of cortisol, compared to partner.

In rodents, endogenous OT influences dopaminergic pathways involved in reinforcement learning. In mice $(74,75)$ and hamsters $(76)$, endogenous OT influences dopaminergic pathways implicated in motivation and reinforcement learning. (77). Here, however, partner touch activated caudate nucleus in the dorsal striatal pathway regardless of OT changes, particularly during the first encounter (Fig2A; 6). This may indicate a general influence on dorsal striatal dopaminergic circuits, biasing behavioral reinforcement. 
Amygdala activation has been widely implicated in human IN-OT studies (44-45,78-81), yet with inconsistent reports of the direction of BOLD changes, implying a dependence on experimental and methodological factors. In mice, OT receptor-expressing neurons in the medial amygdala have been found to mediate olfactory-based social familiarity effects (21). Here, though, stranger touch activated the amygdala in a general fashion (Fig2B; Table s5), supporting the proposal that its prominent role in human and nonhuman primate OT studies may be indirect $(82,83)$.

A specific subtype of $\mathrm{C}$ afferent nerves (C-tactile or CT) have been implicated in affective touch (84-86). CT afferent signaling has been proposed to interact with OT modulation (5). However, we found no evidence for such a relationship; neither somatosensory cortices nor the posterior insular/inferior parietal regions selectively associated with CT stimulation covaried with OT changes, who delivered touch, or in what order. IN-OT has been observed to increase the pleasantness of touch (8), and individuals with higher salivary OT levels have reported greater touch pleasantness (87). Neurons in deep (II-X), but not superficial (I), layers of the spinal cord project to the caudo-dorsal part of the PVN (88), where parvocellular OT neurons are located, and non-peptidergic $\mathrm{C}$ afferent neurons have been observed to express the OT receptor (OXTR; 89). Yet this work has so far not identified OXTR expression in dorsal horn neurons of the spinothalamic tract projecting to the specific thalamic pathways putatively shared by CT afferents $(90,91)$. Further research is therefore needed to dissect the relevant spinothalamic sensory circuitry and its relation to touch-driven OT effects.

The measurement of peripheral OT in humans comes with caveats, as does its relationship with central mechanisms of release. Different methods for detecting plasma OT have yielded different and sometimes uncorrelated sets of value ranges, with measurement issues centering around the selectivity with which testing components detect bound or unbound protein, or whole or fragmentary molecules. Also, cerebrospinal fluid (CSF) has consistently been found to correlate more strongly with brain OT levels than does plasma OT, while CSF and plasma OT measurements have shown weak or no correlation. However, at least a proportion of inconsistencies in reported findings may depend on the investigation of basal levels, in the absence of acute stimuli more likely to evoke coordinated, biologicallymeaningful responses across the central and peripheral nervous systems $(49,93)$, which may also include bioactivity of fragments (93). In this study, within-subject serial sampling 
allowed us to assess evoked OT changes with respect to individual baselines. The covariation of these changes and its temporal patterns with BOLD points to a relationship between the central and peripheral effects of ecologically-evoked endogenous OT, but cannot directly demonstrate it.

In conclusion, these findings offer a methodological and conceptual bridge between stimulus-driven and context-dependent frameworks of endogenous OT modulation of the brain during social interactions. The underlying mechanisms may operate more analogously to a dimmer switch than an "on-off button," changing the gain of present and future hormonal and brain responses. OT's more general role in social interactions may involve reinforcing the status quo with respect to familiar individuals, yet also selectively modulating brain-body responses during interactions with unfamiliar individuals. These findings suggest that such selective differences are modulable across subsequent encounters, and may thus influence neural and behavioral receptivity to touch in social interactions over time. Network hubs such as precuneus and retrosplenial cortex, alongside parieto-temporal pathways, may mediate such influence, turning the "dimmer" of neural processing up or down depending on past and current social context.

Acknowledgments. The authors thank Åsa Axén and Gisela Öhnström for blood sample collection, Kerstin Uvnäs-Moberg, Maria Petersson, Stephanie Preston, and Ellen Lumpkin for valuable discussion, and Paul Hamilton and Irene Perini for assistance with AFNI software. Funding: This study was supported by Distinguished Young Investigator grant FYF-2013-687 from the Swedish Research Council to I.M. Author contributions: I.M. and L.H. designed the study; G.N., L.H., H.L. and I.M collected the data; G.N., L.H., H.L., and R.K. analyzed the data; I.M., G.N., and L.H. wrote the paper. Competing interests: The authors declare no competing interests. Data and materials availability: Anonymized fMRI data are available upon request; blood hormone data availability is limited by ethical, data protection, and materials transfer regulations.

\section{References}

1. Tang, Y., et al. (2020). Social touch promotes interfemale communication via activation of parvocellular oxytocin neurons. Nat Neurosci, 23: 1125-1137. 
2. Kurosawa, M., et al. (1995). Massage-like stroking of the abdomen lowers blood pressure in anesthetized rats: influence of oxytocin. J Auton Nerv Syst, 56: 26-30.

3. Feldman, R. (2012). Oxytocin and social affiliation in humans. Horm Behav 61: 380-391.

4. Uvnäs-Moberg, K., L. Handlin and M. Petersson (2015). Self-soothing behaviors with particular reference to oxytocin release induced by non-noxious sensory stimulation. Frontiers in Psychology 5: 1-16.

5. Walker, S.C. et al. (2017). C-tactile afferents: Cutaneous mediators of oxytocin release during affiliative tactile interactions? Neuropeptides 64, 27-38.

6. Li, Q. et al. (2019). Foot massage evokes oxytocin release and activation of orbitofrontal cortex and superior temporal sulcus. Psychoneuroendocrinol 101, 193-203.

7. Kreuder, A.K. et al. (2017). How the brain codes intimacy: The neurobiological substrates of romantic touch. Hum Brain Mapp, 38, 4525-4534.

8. Chen, Y., et al (2020). Oxytocin increases the pleasantness of affective touch and orbitofrontal cortex activity independent of valence. Eur Neuropsychopharmacol, doi:10.1016/j.euroneuro.2020.08.003

9. M. Mitre et al. (2018). Oxytocin modulation of neural circuits. Curr Top Beh Neurosci 35, 31.53

10. Knobloch, H. S. et al., (2012) Evoked axonal oxytocin release in the central amygdala attenuates fear response. Neuron 73: 553-566.

11. Oettl, L.L. et al. (2016) Oxytocin enhances social recognition by modulating cortical control of early olfactory processing. Neuron 90: 609-621.

12. Burbach, J.P.H. et al (2006). Oxytocin: Synthesis, Secretion and Reproductive Functions. In: Knobil and Neill's Physiology of Reproduction. 3 ed: Elsevier).

13. Qin, J. et al., (2009). Oxytocin receptor expressed on the smooth muscle mediates the excitatory effect of oxytocin on gastric motility in rats. Neurogastroenterol. Motil. 21, 430-438.

14. Filippi, S. et al. (2003). Role of oxytocin in the ejaculatory process. J. Endocrinol. Invest. 26, 82-86.

15. K. Uvnas-Moberg et al. (1998). Oxytocin may mediate the benefits of positive social interaction and emotions. Psychoneuroendocrinol 23, 819-35.

16. Algoe, S.B. et al (2017). Oxytocin and social Bonds: the role of oxytocin in perceptions of romantic partners' bonding behavior. Psychol Sci, 28:1763-1772. 
17. T. Rehn et al. (2014). Dogs' endocrine and behavioural responses at reunion are affected by how the human initiates contact. Physiol Behav, 124, 45-53.

18. Nagasawa, M. et al. (2015). Social evolution. Oxytocin-gaze positive loop and the coevolution of human-dog bonds, Science 348: 333-336.

19. O. Dal Monte, et al (2017). Oxytocin under opioid antagonism leads to supralinear enhancement of social attention. PNAS 114, 5247-5252.

20. Ferretti, V. et al (2019). Oxytocin signaling in the central amygdala modulates emotion discrimination in mice. Curr Biol 17:1938-1953.e6.

21. Ferguson, J.N. et al. (2001). Oxytocin in the medial amygdala is essential for social recognition in the mouse. J. Neurosci. 21, 8278-8285.

22. Quintana D.S., (2020). An allostatic theory of oxytocin. Trends Cogn. Sci. 24: 515-528.

23. J.A. Bartz, et al. (2011). Social effects of oxytocin in humans: context and person matter. Trends Cogn Sci 15: 301-309.

24. J.P. Burkett et al. (2016). Oxytocin-dependent consolation behavior in rodents. Science 351: $375-378$

25. Rickenbacher, E et al (2017). Freezing suppression by oxytocin in central amygdala allows alternate defensive behaviours and mother-pup interactions. Elife 6, doi:10.7554/eLife.24080

26. LoParo, D. et al (2016). Rigorous tests of gene-environment interactions in a lab study of the oxytocin receptor gene (OXTR), alcohol exposure, and aggression. Am J Med Genet B Neuropsychiatr Genet, 171: 589-602.

27. Hovey, D. et al (2016). Antisocial behavior and polymorphisms in the oxytocin receptor gene: findings in two independent samples. Mol Psychiatry 21: 983-988.

28. Anpilov S. et. al. (2020). Wireless Optogenetic Stimulation of Oxytocin Neurons in a Seminatural Setup Dynamically Elevates Both Pro-social and Agonistic Behaviors. Neuron.107: 644655.

29. Huang H. et al (2014). Chronic and acute intranasal oxytocin produce divergent social effects in mice. Neuropsychopharmacology 39: 1102-1114.

30. Leng, G. and Russell, J.A. (2019). The osmoresponsiveness of oxytocin and vasopressin neurones: Mechanisms, allostasis and evolution. J. Neuroendocrinol. 31, e12662.

31. Anacker, A.M. and Beery, A.K. (2013). Life in groups: the roles of oxytocin in mammalian sociality. Front Behav Neurosci 7:185. 
32. De Dreu C.K., et. al. (2010). The neuropeptide oxytocin regulates parochial altruism in intergroup conflict among humans. Science 328: 1408-1411.

33. Heinrichs, M. et al (2003). Social support and oxytocin interact to suppress cortisol and subjective responses to psychosocial stress. Biol Psychiatry 54:1389-98.

34. Di Simplicio, J. (2009). Oxytocin enhances processing of positive versus negative emotional information in healthy male volunteers. Psychopharmacol 23:241-8.

35. Fischer-Shofty, M., et. al. (2010). The effect of intranasal administration of oxytocin on fear recognition. Neuropsychologia 48: 179-184.

36. Petrovic, P. et al (2008). Oxytocin attenuates affective evaluations of conditioned faces and amygdala activity. $J$ Neurosci 28: 6607-6615.

37. Rimmele, U. et al (2009). Oxytocin makes a face in memory familiar. J Neurosci 29: 38-42.

38. Domes, G., et. al. (2007). Oxytocin improves "mind-reading" in humans. Biol Psychiatry 61: 731-733.

39. Ditzen, B., et. al. (2009). Intranasal oxytocin increases positive communication and reduces cortisol levels during couple conflict. Biol Psychiatry, 65(9), 728-731.

40. Scheele, D. et. al. (2012). Oxytocin modulates social distance between males and females. $J$ Neurosci 32: 16074-16079.

41. Scheele, D., et. al. (2013). Oxytocin enhances brain reward system responses in men viewing the face of their female partner. Proc Natl Acad Sci U S A 110: 20308-20313.

42. A.K. Kreuder et al., How the brain codes intimacy: The neurobiological substrates of romantic touch. Hum Brain Mapp, 38, 4525-4534 (2017).

43. Kreuder, A. K. et al (2019). Oxytocin enhances the pain-relieving effects of social support in romantic couples. Hum Brain Mapp, 40: 242-251.

44. D. Wang, et al. (2017). Neural substrates underlying the effects of oxytocin: a quantitative meta-analysis of pharmaco-imaging studies. Soc Cogn Affect Neurosci 12: 1565-73.

45. C.F. Zink \& A. Meyer-Lindenberg, A. (2012). Human neuroimaging of oxytocin and vasopressin in social cognition. Horm Behav 61, 400-9.

46. Striepens, N., et. al. (2013). Elevated cerebrospinal fluid and blood concentrations of oxytocin following its intranasal administration in humans. Sci Rep 3: 3440.

47. Churchland, P. S., \& Winkielman, P. (2012). Modulating social behavior with oxytocin: how does it work? What does it mean? Horm Behav 61: 392-399. 
48. McCullough, M. E. et al (2013). Problems with measuring peripheral oxytocin: can the data on oxytocin and human behavior be trusted? Neurosci Biobehav Reviews 37: 1485-1492.

49. MacLean, E. L., et al (2019). Challenges for measuring OT: The blind men and the elephant? Psychoneuroendocrinology 107: 225-231.

50. Quintana D.S. (2018). Revisiting non-significant effects of intranasal oxytocin using equivalence testing. Psychoneuroendocrinology 87: 127-130.

51. Althammer, F. and Grinevich, V. (2017). Diversity of oxytocin neurons: beyond magno- and parvocellular cell types? J. Neuroendocrinol.

52. Okabe, S. et al (2015). Activation of hypothalamic oxytocin neurons following tactile stimuli in rats. Neurosci Lett 600: 22-27.

53. R. Mottolese et al. (2014). Switching brain serotonin with oxytocin. Proc Natl Acad Sci USA 111: 8637-42.

54. K.M. Igelström et al. (2015). Neural processes in the human temporoparietal cortex separated by localized independent component analysis. J Neurosci 35: 9432-9445.

55. Z.V. Johnson et al., Oxytocin receptors modulate a social salience neural network in male prairie voles. Horm Behav, 87, 16-24 (2017).

56. Shamay-Tsoory S.G. , and Abu-Akel A. (2016). The Social Salience Hypothesis of Oxytocin. Biol. Psychiatry 79: 194-202.

57. Marlin, B.J et al (2015). Oxytocin enables maternal behaviour by balancing cortical inhibition. Nature 520:499-504.

58. Z.V. Johnson et al. (2017). Oxytocin receptors modulate a social salience neural network in male prairie voles. Horm Behav 87: 16-24.

59. Sripada, C. S., et. al. (2013). Oxytocin enhances resting-state connectivity between amygdala and medial frontal cortex. Int J Neuropsychopharmacol 16: 255-260.

60. Martins, D. A. et al (2020). Effects of route of administration on oxytocin-induced changes in regional cerebral blood flow in humans. Nat Commun 11: 1160.

61. Valstad, M. et al (2017). The correlation between central and peripheral oxytocin concentrations: A systematic review and meta-analysis. Neurosci Biobeh Rev 78: 117-124.

62. Pow, D.V. and Morris, J.F. (1989). Dendrites of hypothalamic magnocellular neurons release neurohypophysial peptides by exocytosis. Neuroscience 32: 435-439. 
64. M.L. Seghier (2013). The angular gyrus: multiple functions and multiple subdivisions. Neuroscientist 19: 43-61

63. D.S. Margulies et al (2009). Precuneus shares intrinsic functional architecture in humans and monkeys. PNAS 106: 20069-74.

64. J. Kumar et al. (2019). Oxytocin modulates the effective connectivity between the precuneus and the dorsolateral prefrontal cortex. Eur Arch Psychiatry Clin Neurosci: s00406-019-00989.

65. Petersson, M., \& Uvnas-Moberg, K. (2003). Systemic oxytocin treatment modulates glucocorticoid and mineralocorticoid receptor mRNA in the rat hippocampus. Neurosci Lett 343: 97-100.

66. Vargas-Martinez, F., et. al. (2014). Neuropeptides as neuroprotective agents: Oxytocin a forefront developmental player in the mammalian brain. Prog Neurobiol 123: 37-78.

67. J. Winter \& B. Jurek (2019). The interplay between oxytocin and the CRF system: regulation of the stress response. Cell Tissue Res 375: 85-91.

68. Grewen K.M. et al (2005). Effects of partner support on resting oxytocin, cortisol, norepinephrine, and blood pressure before and after warm partner contact. Psychosom Med 67:531-8.

69. Ditzen, B. et al (2009). Intranasal oxytocin increases positive communication and reduces cortisol levels during couple conflict. Biol Psychiatry 65: 728-731.

70. Etkin, A., Buchel, C., \& Gross, J. J. (2015). The neural bases of emotion regulation. Nat Rev Neurosci 16: 693-700.

71. Davidovic, M., et. al. (2016). Posterior superior temporal sulcus responses predict perceived pleasantness of skin stroking. Frontiers in Human Neuroscience 10: 432.

72. Kaiser, M. D., et. al. (2016). Brain mechanisms for processing affective (and nonaffective) touch are atypical in autism. Cereb Cortex 26: 2705-2714.

73. Voos, A. C., et. al. (2013). Autistic traits are associated with diminished neural response to affective touch. SCAN 8: 378-386.

74. Hung, L.W. et al. (2017). Gating of social reward by oxytocin in the ventral tegmental area. Science 357: 1406-11.

75. Xiao, . L. et al. (2018). Oxytocin functions as a spatiotemporal filter for excitatory synaptic inputs to VTA dopamine neurons. Elife 7, e33892.

76. Song, Z. et al. (2016). Activation of oxytocin receptors, but not arginine-vasopressin V1a receptors, in the ventral tegmental area of male Syrian hamsters is essential for the reward-like properties of social interactions. Psychoneuroendocrinol 74: 164-172. 
77. Groppe et al., S.E. (2013). Oxytocin influences processing of socially relevant cues in the ventral tegmental area of the human brain. Biol Psychiat 74: 172-79.

78. Liu, Y. et al. (2019). Oxytocin modulates social value representations in the amygdala. Nat Neurosci 22: 633-641

79. Motoki, K., et al., (2016). Are plasma oxytocin and vasopressin levels reflective of amygdala activation during the processing of negative emotions? A preliminary study. Front Psychol 7: 480.

80. Sripada, C.S. et al. (2013). Oxytocin enhances resting-state connectivity between amygdala and medial frontal cortex. Int J Neuropsychopharmacol 16, 255-260.

81. Kirsch, P., et. al., Oxytocin modulates neural circuitry for social cognition and fear in humans. J Neurosci, 25(49), 11489-11493 (2005).

82. P.T. Putnam et al. (2018). Bridging the gap between rodents and humans: The role of nonhuman primates in oxytocin research. Am J Primatol 80: e22756.

83. M. Eckstein et al. (2017). Oxytocin differentially alters resting state functional connectivity between amygdala subregions and emotional control networks: Inverse correlation with depressive traits. NeuroImage 149: 458-67.

84. Löken, L. S., et. al. (2009). Coding of pleasant touch by unmyelinated afferents in humans. Nat Neurosci 12: 547-548.

85. Morrison, I. et al (2010). The skin as a social organ. Experimental Brain Research 204: 305314.

86. Olausson, H. et. al. (2002). Unmyelinated tactile afferents signal touch and project to insular cortex. Nat Neurosci 5: 900-904.

87. Portnova, G.V. et al. (2020). Perceived pleasantness of gentle touch in healthy individuals is related to salivary oxytocin response and EEG markers of arousal. Experimental Brain Research, https://doi.org/10.1007/s00221-020-05891-y (epub ahead of print).

88. Gauriau, C., \& Bernard, J. F. (2004). A comparative reappraisal of projections from the superficial laminae of the dorsal horn in the rat: the forebrain. J Comp Neurol 468: 24-56.

89. Nersesyan, Y. et al (2017). Oxytocin Modulates Nociception as an Agonist of Pain-Sensing TRPV1. Cell Rep 21: 1681-1691.

90. Moreno-Lopez, Y. et al (2013). Identification of oxytocin receptor in the dorsal horn and nociceptive dorsal root ganglion neurons. Neuropeptides 47: 117-123. 
91. Andrew, D. (2010). Quantitative characterization of low-threshold mechanoreceptor inputs to lamina I spinoparabrachial neurons in the rat. J Physiol 588: 117-124.

92. Valstad, M.,et al. (2017). The correlation between central and peripheral oxytocin concentrations: A systematic review and meta-analysis. Neurosci Biobehav Rev 78:117-124.

93. Uvnäs-Moberg, K. et. At. (2019). Oxytocin is a principal hormone that exerts part of its effects by active fragments. Med Hypotheses 133: 109394. 